\title{
Research Survey and Research on the Traditional Historical Architecture in Dongcheng District of Beijing
}

\author{
Menglin Xu \\ Nanyang Institute of Technology, Nanyang, China \\ jiumengni @163.com
}

Keywords: Traditional historic buildings; Current situation; Countermeasure and suggestion

\begin{abstract}
Dongcheng District keeps the largest number of historic buildings, the most widely distributed and best-preserved, most feature-rich region buildings in Beijing. The existing historic buildings in Dongcheng District are an important carrier of historical and cultural in the capital, which is also the top priority of the historical and cultural protection in Beijing today. Among these existing historic buildings, there are a large number of them haven't been included in the current system, which still have high preservation value. The department did a thoroughly research about the three cultural relics protection units and research projects census, but didn't do a survey to the historic buildings that except the project, even didn't think about that. Based on information collected in the first historic building survey of Dongcheng District, Beijing. Combining the analysis of historical documents in Dongcheng District, the historic district and traditional historic buildings since the Yuan, Ming, the purpose of this paper is to do an analysis and explore the traditional historic buildings conservation value and protection methods, which also provides a lot of basic information for subsequent protection research.
\end{abstract}

\section{Introduction}

As the world's greatest capitals, Beijing has a strict urban planning at the beginning of Yuan Dynasty. Under the design and construction of Bingzhong Liu and the others, regular chessboard layout of streets, accurate functional partition and intricate but effective water supply systems, have been the indispensable characteristics of Beijing, which also made it famous around the world and be a paragon for capital planning. In Ming and Qing Dynasties, with the development of the inner city which designed on the basis of the pattern that inherited from Yuan dynast, a distinct royal culture characteristic has had becoming to generate. The outer city, however, was different from the inner one. The streets of outer city was built along with the natural lakes and rivers, or depended by the formed terrain, instead of being planned or designed precisely from cover to cover. The business district, besides the above two areas, also showed a special architectural style, which draw a lot of attentions from researchers; the structures built in this area was given strong commercial features regularly, which means that the characteristics of these buildings was peculiar and showed the unique design philosophies of the planners. Above three areas and districts consist the diverse but harmonious city style of Beijing, and thanks to a lot of historical buildings which have been preserved properly, the Dongcheng district of Beijing can also exhibits this special city style up to now.

Dongcheng district is the area with the largest number of historical architecture that is the most widespread with the most complete reservation in Beijing, and it is the important carrier of historical architecture style and traditional culture in Beijing as well as material successor. Also, it is the most important part for the work of Beijing historical and cultural protection.

\section{Definition of Traditional Historical Architecture}

The so-called "historical architecture", is "the buildings with a certain protection value released by the city or county people's government, which can reflect the historical style and features as well as local characteristics, and also are not released for the cultural relic protection unit and not registered for mobile cultural relics". [1] Specially, it includes those traditional buildings and modern 
buildings that have not yet been included in all levels of cultural relic protection units and census register projects, with certain value to protect. As the material carriers that reflect the city's traditional style and historical development process, they are an important part of historical and cultural city. [2]

Traditional historical architecture is a classification of historic buildings, and refers to the buildings with Chinese traditional wooden beam structure or other building materials. It has the largest number in the historic buildings, and is the most widely distributed, with the most complete reservation.

\section{Current Reservation of Traditional Historical Architecture in Dongcheng District of Beijing}

In terms of the distribution, the traditional historical buildings are distributed in all the streets of Dongcheng district in Beijing, but relatively concentrated in the following four streets, namely, Qianmen Street, Jiaodaokou Street, Jingshan Street, Chaoyangmen Street. For example, among more than seven hundred traditional historical buildings in the survey, the traditional historical buildings in Qianmen street account for $55 \%$ of the total number.

From the perspective of the courtyard pattern, for the layout of street and courtyard in the north area (Chang'an avenue as a boundary to divide the north area and the south area), it mainly includes the standard buildings inheriting from Yuan, Ming and Qing Dynasty, and there are a fewer standard Siheyuan in the south area, with the flexible layout.

From the point of building function, in Dongcheng district, it has the most housing construction, which is the most widespread with the most time span. This kind of buildings includes large palace as well as general residential in terms of size; and in the light of construction age, they were constructed in various periods in Ming and Qing Dynasty and even after the founding of the nation. Religious buildings, Yamen, government agencies are mainly concentrated in the north area, and the halls, commercial buildings are mainly concentrated in the south area. Warehouse, hospital, school are scattered in the streets.

In terms of the current situation of reservation, better reserved traditional historical buildings account for $13 \%$ of the total number in this investigation; those with general reservation account for $87 \%$. The traditional historical buildings in the north area account for $40 \%$; those in the south area account for $60 \%$; the number of the south area is $20 \%$ more than that of the north south, which is because the survey of the north area is relatively profound in the investigation of the historic buildings in the past years, and the survey of the north area relatively lacks the focus on the historical buildings; so in the survey, the number in the south area is one fifth more than that in the north.

In addition, for the better reserved traditional historical buildings, the number in the north area is obviously more than that in the south; for its reason, the buildings are mostly the housing for dignitaries in the north area, while a lot of people with free occupation live in the south area, so the traditional buildings in the south area cannot compete with those in the north area in terms of quality, volume, etc.

\section{Current Problems to Traditional Historical Architecture in Dongcheng District of Beijing}

Insufficient Recognition of the Special Value of Traditional Historical Architecture. As can be seen in the survey, because of more reserves in the north area, and the traditional architectural specification in this area is high, so the reservation of the traditional buildings in the north of Chang'an Street is relatively superior to that in the south. But unlike the north area, because of the special city function orientation and spontaneous formation process, it makes the historical buildings in the south area not obviously very neat, and most of valuable buildings have small scale with the irregular specification. Based on the standard of the north area, it seems to have lower grade. However, unlike "standardized" courtyard in the north area, it is the irregular features that give the valuable traditional historical buildings in the south with individuality, variety and non-replication, and each group of buildings is not the same individual, and has the rich 
individuality, which is the biggest value and charm. As a witness of a special period in Chinese history, the free commercial atmosphere in the south and the full-bodied life atmosphere in the north complement each other; upon the protection, we must fully consider the different characteristics and value, and cannot ignore anyone of them. [3]

However, there was the lack of recognition of this unique personality and positioning for the traditional architecture in the south area in the past years. Its irregular features is one-sided understood as the characteristics of the old, low level, poor protection value; when judging the value of historical building protection, the standard for the north area is completely copied. As a result, on the macro level, in the south area, the historical and cultural reserve area is only just one place with the size of the fish month; on the micro level, in terms of the specified protective measures, some buildings with the most protection value are often ignored.

Insufficient Survey and Research. In addition that there are relatively detailed investigations on the static cultural relics of all kinds in the north of Dongcheng district, there is the lack for the special investigation of the historical buildings in the other old areas in Beijing, and the researches on the old buildings are mostly combined with the projects such as cultural relics census, and do not involve the renovated demolition areas; also, the management department lacks in-depth and quantitative knowledge for the present situation of the historical buildings within the respective jurisdictions, and this problem is more serious in the south of Dongcheng district. When the public cannot be fully aware of the current historical building value, under the background of urbanization in Beijing that cannot be $t$ suppressed, Hutong and Siheyuan greatly disappear. This delays and hinders the issuing of the protection policy, and more directly causes that some historical buildings have disappeared or are about to disappear before their values are fully known and discovered.

Therefore, this research timely makes up for the defect, covering the problems in the previous investigations, and ending the situation that there is lack of special research of historical buildings in Beijing, with large innovative and demonstration value.

Lack of Target Policy and Protection Measure. As mentioned above, due to being not included in the current system for the protection of cultural relics, there is the lack of the corresponding mandatory protection norms and measures with operability for historic buildings, causing all sorts of demolition and reconstruction behavior become the biggest threat at present. Related departments have no rule to follow in the face of save the historical buildings that will be demolished, thereby delaying the protection. [4]

The demolition and reconstruction behavior for historical buildings found in this survey is arranged based on the damage severity as follows:

First, dangerously renovated demolition

The golden fish pond and Chongwenmen street that have been renovated are taken as representative, their damage is comprehensive and devastating; not only historic buildings but also the historical block will disappear.

At present, Beijing's renovated demolition takes commercial land development pattern, and the land needs to be transacted in market, even if it is the historical culture protection area that has already announced. And according to the current laws and regulations, "clear" must be done before the land enters in market, so in addition to the individual cultural relic protection units, a large number of historical buildings will be demolished. In a sense, the overlapping of the published renovated demolition area and historical culture protection area is a tragedy.

Second, dangerous house renovation

It specifically means the housing renovation presided by a street or housing management office based on the original site to distinguish it from renovated and self-built residents. The harm degree is inferior to the renovated people, but is still likely to cause serious damage to historical buildings. It is reflected that during dismantling the old and constructing the new, although it objectively improves the residents' living environment, the building structure, form, practice after the renovation is already out of the original system, without cultural value; in addition, in the governance of the environment in some streets, the historical building's exterior wall is paint with lagging processing, which also has a great influence on the appearance and the integrity. 
Third, self-reconstruction and expansion by working unit or individual

It means the working units or individuals reconstruct and expand the historical buildings in order to repair the house, expand the use space, construction change, etc. Compared with the first two, this kind of behavior has relatively small damage for the destruction of historical buildings, and is limited to a single building. However, companies and foreign personnel to take the complete reconstruction after buying it, which can make the protection value of historical buildings disappear completely; and this kind of behavior can be seen everywhere, with large quantity, thus, the destruction is serious with a large scale.

\section{The Solutions to Traditional Historical Architecture in Dongcheng District of Beijing}

For the above three problems, the following strategies and suggestions are put forward.

Introducing Advanced Protection Concept . When changing the static cultural relics protection in the past, the protection method of single piece is preferred, introducing advanced protection concepts, and the historical block is regarded as a whole; the protection units at all levels, census register projects and historic buildings are combined without neglecting anyone, together constituting the static protection system of cultural relics. At the same time, the shift in protection mode should be made, avoiding the large-scale replacement of inhabitants for the historical and cultural blocks, in order to maintain long-term formed folkway and folk custom, and protect the integrity of the historic blocks in terms of the traditional style.

In the repair and protection of historic buildings, we should follow the three principles of the modern cultural relics protection technology, that is, minimum intervention, reversible and identifiable, only to repair the part that endangers the safety of the building, to try to use the raw material, and at the same time to make a clear distinction between repaired and unrepaired parts; it should ban the unified paint, lagging and other destructive and invasive street beautification for the historical buildings, in order to maximize the preserved primitive simplicity and charm of the historical buildings. [5]

Establishing and Perfecting the Current Archive of Historical Architecture. As the basic part of historical building protection, on the basis of investigation and research, it should establish a relatively perfect architectural archives system, with the following three important significances:

First, in view of the serious situation of historical building protection at present stage, in the short term, when the effective protection means cannot be taken, through the establishment of all-round and three-dimensional archives, it can provide the basis for the repair or reconstruction in the future, which must become the priority of work.

Second, on the basis of the establishment of archive system, it should have the overall understanding on the current situation of the protection of historic buildings in the area, to make scientific and feasible s definition standards and evaluation indexes for the historical building, and based on the above criteria defined scope of protection. The scope of protection should clearly point out the part with the historical buildings that must be kept, and the part that can be demolished; for the sections that need to retain, the clear specific statement should be made to point whether we only need to keep building structure, or protect it along with the original decoration, etc., in order to emphasize practical operation. For some historic buildings with important protection value, it should also hang the instruction sign to propaganda historical building protection concept to the public.

Third, it should fully research, and make details about the archive establishment, which are also the premise for formulating scientific and reasonable, feasible protection policies and regulations.

Issuing the Special Protection Policy and Regulation. For historical buildings, special policies and regulations should be issued, and included in the current system for the protection of cultural relics, which is the premise and foundation for effective protection. Only in the form of law to determine the historic nature of cultural relics can the damage to its behavior will be under the constraints and limitations of relevant laws and regulations for the protection of cultural relics, so that the effective accountability system can be set up, and the historical building protection is truly practice by following the laws. 
The policies and regulations should emphasize the following two parts:

First, there is clear responsibility division. Planning and cultural relics department have the rights and obligations for the supervision of historic buildings. It should designate staff or community volunteers, supervisors to regularly patrol, conduct sampling inspection of historical building preservation and usage in the jurisdiction, timely stop unreasonable use and destruction; it shall provide scientific guidance on the use, repair and maintenance of historic buildings within the jurisdiction area, and provide training for the users and owners of the historical architecture on how to identify protection scope, how to reasonable use and maintain, how to stop and complain destruction; it should make use of media, theme preach, display panel, issuing folding or manual, etc. to spread the related knowledge on the historical building protection to the public in a timely manner; it should introduce and popularize the use of domestic and foreign advanced protection concepts and management concepts, etc.

In addition, it is suggested to add the responsibility planners and architects to work full-time in charge of technical protection of historical buildings.

Second, it should set up the accountability system. Based on specific rights and obligations of both sides, practical and effective system of accountability should be made with strict punishment for the severe destruction of historic buildings to avoid similar situations.

Given the accountability system has not yet been established, through publicity and education, it should establish incentive system to improve the consciousness of the units with property right and the users on historical building protection, and change passive into active protection, which is the most effective way to avoid related vandalism.

Further Improving Residents' Eager and Important Recognition for the Protection of Siheyuan. Siheyuan is the basic unit to constitute the old districts of Beijing, with high historical value and cultural value. At present, because the residents lack recognition for it, it has caused varying degrees of destruction of historical buildings. In the investigation of traditional architecture, nearly half of the courtyards are destructed because of private building or dismantling, therefore, we should improve residents' consciousness of the protection of Siheyuan, which is the key to protect the traditional historical architecture.

\section{Conclusion}

Dongcheng district has the largest number of historic buildings, and it was the most widely distributed, the original ecology preserved very well. Traditional historical buildings is constitute the basic unit of the city -hutong-courtyard, and it has an important significance for the research. In this paper, the analysis and evaluation is relatively shallow, many theoretical analysis is not enough in-depth, but we still hope that through the existing condition of traditional historical buildings in dongcheng district in Beijing, we can provide some advice about the future protection and quantitative evaluation for the government.

\section{References}

[1] The Regulations on Protecting Historical Famous Towns and Villages.

[2] Z.X.Zhu, The Planning and the Cultural Origin of the Ancient Beijing, The Chinese Publishing House, Beijing, 2007.

[3] R.Z.Hou. THE Historical Geography of Beijing, Yanshan Publishing House, Beijing, 2000 pp. 150-151.

[4] Y.X.Deng. Courtyard House in Beijing, People's Daily Publishing Corporation, Beijing, 1990.

[5] J.F.Zhu. Sixty Years of Chinese Architecture (1949-2009): History, Theory and Criticism, China Building Industry Press, Beijing, 2009. 